\title{
Phase behavior and formation of oleyl ester nanoemulsions system
}

\begin{abstract}
Oleylesters (OEs) are newly synthesized esters from different fatty acids of different chain length and oleyl alcohol using Novozyme 435 as catalyst. The phase behavior of these esters were determined by constructing the pseudoternaryphase diagrams of OEs/Tween 80/water at $25.0 \pm 0.5^{\circ} \mathrm{C}$. Compositions from the isotropic and homogeneous region were selected for characterization. The shortest OEs chain represents the most stable nanoemusions system with the smallest droplet for both isotropic and homogeneous regions. The results from simulation study showed that the shape of emulsion droplet was in spherical shape with the values of eccentricity (e) 0.11 to 0.17 .
\end{abstract}

Keyword: Nanoemulsions; Oleylester; Pseudoternary phase diagram; Tween 80 